\title{
Pericardial effusion in patients with cancer: outcome with contemporary management strategies
}

Roger J Laham, David J Cohen, Richard E Kuntz, Donald S Baim, Beverly H Lorell, Michael Simons

\begin{abstract}
Objective-To investigate the clinical presentation and current management strategies of pericardial effusion in patients with malignancy.

Design-Retrospective single centre, consecutive observational study. Setting-University hospital.

Patients-93 consecutive patients with a past or present diagnosis of cancer and a pericardial effusion, including 50 with a pericardial effusion $>1 \mathrm{~cm}$.

Results-Of the 50 patients with pericardial effusions $>1 \mathrm{~cm}$, most had stage 4 cancer $(64 \%)$, were symptomatic at the time of presentation (74\%), and had right atrial collapse $(74 \%)$. Twenty patients were treated conservatively (without pericardiocentesis) and were less symptomatic $(55 \% \quad v \quad 87 \%, P=0.012)$, had smaller pericardial effusions $(1.5(0.4) v$ $1.8(0.5), P=0.02)$, and less frequent clinical $(10 \% v 40 \%, P=0.02)$ and echocardiographic evidence of tamponade $(40 \% v$ $97 \%, P<0.001)$ than the 30 patients treated invasively with initial pericardiocentesis $(n=29)$ or pericardial window placement $(n=1)$. Pericardial tamponade requiring repeat pericardiocentesis occurred in $18(62 \%)$ of 29 patients after a median of 7 days. In contrast, only four $(20 \%)$ of 20 patients in the conservative group progressed to frank clinical tamponade and required pericardiocentesis ( $P=0.005 v$ invasive group). The overall median survival was 2 months with a survival rate at 48 months of $26 \%$. Survival, duration of hospital stay, and hospital charges were similar with both strategies. By multivariable analysis, the absence

and should be considered for initial treatment.

(Heart 1996;75:67-71)

Keywords: pericardial effusion; pericardial tamponade; cancer

The management of pericardial effusions in patients with malignancy is a difficult problem that confronts the oncologist and the cardiologist. Postmortem series of patients with cancer reveal metastases to the heart and pericardium in about $15-20 \%$ of patients. ${ }^{1-6}$ The most common manifestation of secondary cardiac metastasis is pericardial effusion in $5-15 \%$ of patients with cancer. ${ }^{67}$ In addition, non-malignant pericardial effusion may be found in as many as $7 \%$ of patients with cancer at postmortem examination. ${ }^{78}$

Despite the magnitude of this serious problem there has been little progress in the management of pericardial effusions in patients with cancer over the past decade, and no approach for their treatment has been universally accepted. ${ }^{9}$ Possible management strategies include observation with intensified chemotherapy or radiation treatment, catheter pericardiocentesis, pericardiotomy, or pericardiectomy. Furthermore, pericardial effusion is diagnosed before clear symptoms develop in many patients with cancer by more frequent use of surveillance echocardiography. ${ }^{9}$ We undertook this retrospective study to investigate the clinical presentations, as well as current diagnostic and management strategies of pericardial effusion in patients with malignancy.
\end{abstract} of symptoms was the only independent predictor of long-term survival (relative hazards ratio $=3 \cdot 2, P=0.05$ ). Survival was similar in the 43 patients with cancer and pericardial effusions of $\leqslant 1 \mathrm{~cm}$.

Conclusion-Asymptomatic patients with cancer and pericardial effusion can be managed conservatively with close follow up. In patients with symptoms or clinical cardiac tamponade, pericardiocentesis provides relief of symptoms but does not improve survival and has a high recurrence rate. Surgical pericardial windows or possibly percutaneous balloon pericardiotomy should be used for recurrences

\section{Patients and methods}

STUDY POPULATION

Between 1986 and 1992, 93 consecutive patients who had a past or present diagnosis of cancer and an echocardiogram showing pericardial fluid accumulation were identified. Forty three patients had a small $(<1 \mathrm{~cm})$ pericardial effusion. The remaining 50 patients had a pericardial effusion $>1 \mathrm{~cm}$ in thickness and were the focus of this study. The hospital charts and echocardiographic findings were reviewed to determine the presenting characteristics, management strategies, and clinical outcomes. 
CLINICAL EVALUATION

All patients were interviewed and examined by a cardiologist. The combination of a paradoxical pulse greater than $12 \mathrm{~mm} \mathrm{Hg}$, a jugular venous pressure greater than $10 \mathrm{~mm} \mathrm{Hg}$, and/or hypotension (systolic blood pressure $<100 \mathrm{~mm} \mathrm{Hg}$ ) was considered diagnostic criteria for cardiac tamponade. Standard $M$ mode and two dimensional echocardiography were used to determine the size of the pericardial effusion and the presence of right atrial and ventricular collapse. ${ }^{10-12}$

PERICARDIOCENTESIS AND PERICARDIAL WINDOW Right sided cardiac catheterization was performed with all pericardiocenteses. Pericardiocentesis was performed via the subxyphoid approach using a soft multihole catheter advanced to the pericardial space over a J tipped guidewire. After initial vacuum drainage, the catheter was left in for $24 \mathrm{~h}$ for further closed gravity drainage. ${ }^{10}$ The pericardial fluid was analysed by complete blood count, determination of glucose and protein levels and cytological examination. Pericardial windows were performed surgically by the subxyphoid approach.

Table 1 Baseline clinical characteristics

\begin{tabular}{ll}
\hline & Patients $(n=50)$ \\
\hline Mean (SD) age (years) & $53(17)$ \\
Sex (M:F) & $13: 37$ \\
Cancer stage (\%) & 36 \\
$1-3$ & 64 \\
4 & $18(36)$ \\
Previous radiation treatment (\%) & $21(24)$ \\
Interval to pericardial effusion (months) & $37(74)$ \\
Symptoms (\%) & $33(66)$ \\
Dyspnoea & $15(30)$ \\
Chest pain & $14(28)$ \\
Paradoxical pulse $\geqslant 12$ mm Hg (\%) & $12(24)$ \\
Jugular venous pressure > 10 mm Hg (\%) & $2(4)$ \\
SBP < 100 mm Hg (\%) & $4(8)$ \\
Rub ${ }^{\star} \%$ ) & $28(56)$ \\
Cardiomegaly (\%) & $21(42)$ \\
Pleural effusion (\%) & $36(72)$ \\
ECG abnormalities (\%) & $36(72)$ \\
Sinus tachycardia (\%) & $8(16)$ \\
Low voltage (\%) & $2(4)$ \\
ST segment abnormalities (\%) & $1 \cdot 7(0 \cdot 4)$ \\
Mean (SD) effusion size (cm) & $37(74)$ \\
Right atrial collapse (\%) & $26(52)$ \\
Right ventricular collapse (\%) & \\
\hline SBP, systolic blood pressure; ECG, electrocardiogram. \\
^Pericardial friction rub.
\end{tabular}

Table 2 Clinical characteristics of non-invasive versus invasive group

\begin{tabular}{lccl}
\hline & $\begin{array}{c}\text { Non-invasive group } \\
(n=20)\end{array}$ & $\begin{array}{c}\text { Invasive group } \\
(n=30)\end{array}$ & $P$ value \\
\hline Mean (SD) age (years) & $53(16)$ & $53(18)$ & NS \\
Sex (M:F) & $4 / 16$ & $9 / 21$ & NS \\
Mean (SD) cancer stage 1-4 & $3 \cdot 4(0 \cdot 8)$ & $3 \cdot 8(0 \cdot 5)$ & NS \\
& $($ median $=4)$ & $($ median $=4)$ & \\
Previous radiation treatment (\%) & $6(30)$ & $12(40)$ & NS \\
Mean (SD) interval to pericardial effusion & $16(18)$ & $24(27)$ & NS \\
(months) & $11(55)$ & $26(87)$ & 0.012 \\
Symptoms (\%) & $6(30)$ & $27(90)$ & 0.052 \\
Dyspnoea & $5(25)$ & $10(33)$ & NS \\
Chest pain & $2(10)$ & $12(40)$ & 0.02 \\
Paradoxical pulse $\geqslant 12$ mm Hg (\%) & $2(10)$ & $12(40)$ & $<0.001$ \\
Jugular venous pressure $>10$ mm Hg (\%) & 0 & $2(7)$ & NS \\
SBP 100 mmHg (\%) & $4(20)$ & $24(80)$ & $<0.001$ \\
Cardiomegaly (\%) & $8(40)$ & $13(43)$ & NS \\
Pleural effusion (\%) & $13(65)$ & $23(77)$ & NS \\
Positive ECG (\%) & $13(65)$ & $23(77)$ & NS \\
Sinus tachycardia & $1(5)$ & $7(23)$ & NS \\
Low voltage & 0 & $2(7)$ & NS \\
ST segment abnormalities & $1 \cdot 5(0 \cdot 4)$ & $1 \cdot 8(0.5)$ & 0.02 \\
Mean (SD) pericardial effusion size (cm) & $8(40)$ & $29(97)$ & $<0.001$ \\
Right atrial collapse (\%) & $3(15)$ & $23(77)$ & $<0.001$ \\
Right ventricular collapse (\%) & &
\end{tabular}

SBP, systolic blood pressure; ECG, electrocardiogram; NS, not significant.

\section{FOLLOW UP}

Clinical follow up consisted of chart review and interview of primary physicians. Clinical follow up (of at least 6 months or time to death) was available for all patients averaging 10 (13) (range 0-48) months. Survival was calculated from the date of initial positive echocardiogram to the date of death or last follow up.

\section{STATISTICAL METHODS}

Data are expressed as mean (1 SD). Continuous variables were compared by unpaired Student's $t$ test, while categorical variables were compared by $\chi^{2}$ analysis. Survival was estimated by Kaplan-Meier curves, and subgroups were compared using the log rank test. ${ }^{13} 14$ The independent association between demographic, clinical, echocardiographic, and haemodynamic findings and survival was determined by stepwise regression using the Cox proportional hazards model. ${ }^{15}$ All $p$ values were two tailed and a $p$ value $\leqslant 0.05$ was considered significant.

\section{Results}

The study cohort comprised 50 patients (13 men and 37 women); mean (SD) age 53 (17) years with pericardial effusions $>1 \mathrm{~cm}$ making them eligible for pericardiocentesis (with $1 \mathrm{~cm}$ representing the minimum amount of pericardial fluid required for safe pericardiocentesis at our institution). Table 1 summarises the baseline clinical characteristics of these patients. Sixteen patients had non-small cell lung cancer, 11 breast cancer, 10 lymphoid malignancies (four with Hodgkin's disease and six with non-Hodgkin's lymphoma), and 13 other malignancies. Most patients were symptomatic at the time of presentation: 33 patients $(66 \%)$ had dyspnoea and $15(30 \%)$ chest pain. Fourteen patients $(28 \%)$ had a paradoxical pulse > $12 \mathrm{~mm} \mathrm{Hg}, 12(24 \%)$ a jugular venous pressure $>10 \mathrm{~mm} \mathrm{Hg}$, but only two (4\%) were hypotensive.

Of the 50 patients with moderate or large pericardial effusions, 20 were treated conservatively with serial clinical and echocardiographic follow up and represent the non-invasive group. The remaining 30 patients were treated with initial pericardiocentesis (29 patients) or pericardial window placement (one), and constitute the invasive group. The decision whether to treat patients conservatively or invasively was made by the consulting cardiologist without any predefined protocol. Patients in the non-invasive group were less commonly symptomatic $(55 \%$ v $87 \%, P=0.012$ ), less likely to have features of clinical cardiac tamponade $(10 \% \quad v \quad 40 \%$, $P=0.02$ ), had smaller pericardial effusions $(1.5(0.4) \mathrm{cm} v 1.8(0.5) \mathrm{cm}, \mathrm{P}=0.02)$, and were less likely to have echocardiographic evidence of right atrial $(40 \% v 97 \%, \mathrm{P}<0.001)$ or right ventricular $(15 \% v 77 \%, \mathrm{P}<0.001)$ collapse than patients in the invasive group (table 2). Four of the 20 patients treated noninvasively progressed to frank clinical cardiac tamponade, and required pericardiocentesis after a mean (SD) of 41 (16) days from initial 
Table 3 Immediate pericardiocentesis group: recurrence versus no recurrence

\begin{tabular}{lcc}
\hline & $\begin{array}{l}\text { Recurrence } \\
(n=18)\end{array}$ & $\begin{array}{l}\text { No recurrence } \\
(n=11)\end{array}$ \\
\hline Mean (SD) age (years) & $54(16)$ & $53(19)$ \\
Men (\%) & $4(22)$ & $5(45)$ \\
Mean (SD) cancer stage 1-4 & $3 \cdot 9(0 \cdot 3)$ & $3 \cdot 5(0 \cdot 8)$ \\
& $($ median $=4)$ & $($ median $=4)$ \\
Prior radiation treatment (\%) & $6(33)$ & $6(55)$ \\
Mean (SD) pericardial pressure (mm Hg) & $15 \cdot 1(4 \cdot 2)$ & $14 \cdot 0(4 \cdot 5)$ \\
Mean (SD) RA pressure (mm Hg) & $15 \cdot 5(5 \cdot 0)$ & $15 \cdot 3(5 \cdot 0)$ \\
Mean (SD) post-tap RA pressure (mm Hg) & $8 \cdot 2(4 \cdot 5)$ & $9 \cdot 4(5 \cdot 0)$ \\
Positive cytology (\%) & $10(56)$ & $5(45)$ \\
\hline
\end{tabular}

Differences are not significant. RA, right atrial.

Table 4 Long-term clinical outcomes by treatment groups

\begin{tabular}{|c|c|c|c|}
\hline & $\begin{array}{l}\text { Non-invasive group } \\
(n=20)\end{array}$ & $\begin{array}{l}\text { Invasive group } \\
(n=30)\end{array}$ & $P$ value \\
\hline Hospital mortality (\%) & $2(10)$ & $6(20)$ & NS \\
\hline Mean (SD) follow up duration (months) & $11(14)$ & $10(12)$ & NS \\
\hline Overall mortality (\%) & $15(75)$ & $22(73)$ & NS \\
\hline Progression or recurrence (\%) & $4(20)$ & $18(60)$ & $<0.005$ \\
\hline $\begin{array}{l}\text { Mean (SD) duration of initial } \\
\text { hospitalization (days) }\end{array}$ & $17(15)$ & $17(13)$ & NS \\
\hline Mean (SD) hospital charges & $\mathrm{n}=14$ & $\mathrm{n}=9$ & \\
\hline Initial & $21530(19500)$ & $32440(39630)$ & NS \\
\hline Follow up & $32280(28840)$ & $44750(17650)$ & NS \\
\hline Overall & $42280(28790)$ & $77190(46590)$ & NS \\
\hline
\end{tabular}

NS, not significant. decreased to $0 \mathrm{~mm} \mathrm{Hg}$ and the mean right atrial pressure decreased to $9(5) \mathrm{mm} \mathrm{Hg}$ (median $9 \mathrm{~mm} \mathrm{Hg}$ ) after pericardial drainage. Fluid cytology was positive for malignant cells in 15 patients $(52 \%)$. The pericardial effusion recurred in $18(62 \%)$ of 29 patients with echocardiographic signs of cardiac tamponade necessitating repeat intervention (pericardiocentesis in two patients $(11 \%)$ and pericardial window placement in $16(89 \%)$. The mean (SD) time to recurrence was 45 (71) days with a median of 7 days. One of the 18 patients, who had a pericardial window after his first recurrence, had a second recurrence after 12 months.

Patients in the immediate pericardiocentesis group with or without recurrence of the pericardial effusion, did not differ significantly in their baseline clinical characteristics, pericardiocentesis findings, or clinical course (table 3 ). No predictor of recurrence of the pericardial effusion could be identified.

Only one $(69 \%)$ of 18 patients who underwent pericardial window placement (one as initial treatment and 17 as treatment of recurrent pericardial effusions) had a recurrence of pericardial effusion after 12 months. This is much lower than in patients treated with pericardiocentesis in whom pericardial effusion recurred in 19 of 33 patients (18 of 29 in the invasive group and one of four in the noninvasive group, $P=0.001$ ).

LONG-TERM FOLLOW UP

At the conclusion of the study (after a mean (SD) follow up of 10 (13) months), 37 (74\%) of the 50 patients had died (table 4, fig). Pericardial effusion was the immediate cause of death, however, in only one patient (as a result of right ventricular puncture during pericardiocentesis). Median survival was 2 months with a long-term (48 months) survival rate of $26 \%$. Survival was similar in both groups.

All variables were analysed to determine those that correlated with survival. In univariable analysis, the absence of symptoms (relative hazards ratio $=2 \cdot 9, P=0.02)$ and sinus tachycardia at presentation (relative hazards ratio $=3 \cdot 6, P=0.007$ ) were the only predictors of survival. The only independent predictor of long-term survival with multivariable analysis was the absence of symptoms at initial presentation (relative hazards ratio $=3 \cdot 2$, $P=0.05)$.

When all 93 patients (patients with cancer and any pericardial effusion) were included in the study (table 5), the mortality was $70 \%$

Table 5 Characteristics of all 93 patients with cancer and pericardial effusion

\begin{tabular}{ll}
\hline & $\begin{array}{c}\text { Patients } \\
(n=93)\end{array}$ \\
\hline Mean (SD) age (years) & $57(18)$ \\
Men (\%) & $30(32)$ \\
Symptoms (\%) & $62(66)$ \\
Mean (SD) size of pericardial effusion (cm) & $1 \cdot 2(0 \cdot 7)$ \\
Overall mortality (\%) & $65(70)$ \\
Survival (months) & \\
$\quad$ Mean & 11 \\
$\quad$ Median & 4 \\
\hline
\end{tabular}


with a mean survival of 11 months and a median survival of 4 months. In 45 patients with pericardial effusions of $\leqslant 1 \mathrm{~cm}$, only two (4\%) had progression of their pericardial effusion to $>1 \mathrm{~cm}$ in size. In univariable analysis, the absence of symptoms remained the only predictor of long-term survival $(P=0.011)$.

\section{Discussion}

Pericardial effusions are detected with increasing frequency in patients with malignancy by greater use of surveillance echocardiography. Once pericardial effusion is diagnosed, the complex management of the patient with cancer is further clouded by questions regarding the need for pericardial drainage versus close observation with serial clinical evaluation and echocardiography. We carried out a retrospective analysis of our experience with pericardial effusion in patients with cancer in an attempt to determine the optimum management strategy in these patients.

Not surprisingly, survival was poor in this series of 93 consecutive patients with cancer and pericardial effusion. Most patients (96\%) with pericardial effusions of $\leqslant 1 \mathrm{~cm}$ did not have progression of their pericardial effusion. More importantly, we found that a non-invasive treatment strategy in patients with pericardial effusions $>1 \mathrm{~cm}$ but without clinical tamponade did not seem to increase mortality, lengthen hospital stay, or increase cost in either the short or long term (fig, table 4). By contrast, immediate pericardiocentesis, carried out in 29 patients, while providing symptomatic relief in all cases, resulted in a high rate of recurrence $(62 \%)$ that could not be predicted reliably from the baseline clinical, echocardiographic, or haemodynamic findings. In contrast, only one of the 18 patients who underwent creation of a subxyphoid pericardial window had a recurrence of pericardial effusion, which was significantly lower than the recurrence rate after pericardiocentesis.

The median survival time of our patients was 120 days, which was higher than the 80 days reported in some series ${ }^{16-19}$ but lower than the 170 days reported in another. ${ }^{9}$ This discrepancy may be related to the small number and the different types of malignancy in various studies. In our series, pericardial fluid contained malignant cells in $52 \%$ of patients who underwent pericardiocentesis; this finding is similar to the results of some investigators ${ }^{7}$ but discrepant with others. ${ }^{20-22}$ One patient died as a complication of pericardiocentesis giving a procedural mortality of $2 \cdot 7 \%$ (total of 37 pericardiocenteses including the non-invasive group). This value is lower than in previous studies reporting a complication rate of $10-25 \%$ and that of clinically significant ventricular laceration of $7 \% . .^{22}{ }^{23}$ The recurrence rate of pericardial effusion after successful pericardiocentesis in our series was $58 \%$ (including all pericardiocenteses), which is higher than the $13-50 \%$ reported in most series. $^{22-27}$ This difference may be related to the higher cancer stage, and complete clinical and echocardiographic follow up in our series.
The recurrence rate after subxyphoid pericardial window placement $(6 \%)$ was within the range $(0-12 \%)$ reported in the literature. ${ }^{19} 2728$

There are several limitations to this study including the retrospective study design, the small sample size, the heterogeneous patient population, and the arbitrary division of the study sample into various groups. These limit the applicability of the results to the general population.

\section{CLINICAL IMPLICATIONS}

Consensus regarding the management of pericardial effusion in patients with malignancy is not established. Because of the small number of patients in every series including ours, the heterogeneity of the study population, the concomitant use of multiple treatment modalities, and varying criteria for a clinical response, stringent recommendations are not possible. Any treatment plan should take into consideration the need to diagnose cancer relapse, the patient's life expectancy, symptomatic status, as well as the radiosensitivity and chemosensitivity of the tumour.

The data presented in this study nonetheless suggest that asymptomatic patients with pericardial effusions regardless of their size should be managed with close follow up and, if possible, treatment of the underlying malignancy. Pericardiocentesis or more preferably surgical pericardial window should be performed only for symptoms or the need for diagnosis. An alternative treatment is pericardiocentesis with pericardial sclerosis, a strategy not used in this patient population. ${ }^{29}$ In patients with symptoms or clinical cardiac tamponade and haemodynamic compromise, emergency pericardiocentesis provides efficient relief of symptoms but does not improve overall survival. A surgical pericardial window should be used for recurrence and should be seriously considered as an initial intervention given the high recurrence rate associated with catheter pericardiocentesis. Percutaneous techniques for window formation by balloon dilatation of the pericardium at the time of initial pericardiocentesis may ultimately offer some advantage for these patients. ${ }^{27} 280^{-32}$

1 Appelqvist P, Maamies T, Grohn P. Emergency pericardiotomy as primary diagnostic and therapeutic procedure diotomy as primary diagnostic and therapeutic procedure and review of the literature. F Surg Oncol 1982;21:18-22.

Biran S, Hochman A, Levij IS, Stern S. Clinical diagnosis of Biran S, Hochman A, Levij IS, Stern S. Clinical diagnosis of
secondary tumors of the heart and pericardium. Dis Chest 1969;55:208-18

3 Lockich JJ. The management of malignant pericardial effusions. $\mathscr{F} A M A$ 1973;224:1401-4.

4 Piehler JM, Pluth JR, Schaff HV, et al. Surgical management of effusive pericardial disease. $\mathcal{F}$ Thorac Cardiovas Surg 1985;90:506-16.

5 Gregory JR, McMurtrey MJ, Mountain CF. A surgica approach to the treatment of pericardial effusion in cance patients. Am 7 Clin Oncol 1985;8:319-23.

6 Cham WC, Freiman AH, Carstens HB, Chu FCH Radiation therapy of cardiac and pericardial metastasis. Radiology 1975;114:701-4.

7 Posner MR, Cohen GI, Skarin AT. Pericardial disease in patients with cancer. Am $\mathcal{F}$ Med 1981;71:407-13.

8 Agner RC, Gallis HA. Pericarditis. Differential diagnostic considerations. Arch Intern Med 1979;139:407-12.

9 Buck M, Ingle JN, Giuliani ER, Gordon JR. Therneau TM. Pericardial effusion in women with breast cancer. Cancer 1987;60:263-9.

10 Levine MJ, Lorell BH, Diver DJ, Come PC. Implications of echocardiographically assisted diagnosis of pericardial tamponade in contemporary medical patients: detection before hemodynamic embarrassment. I Am Coll Cardio 1991;17:59-65. 
11 Singh S, Wann LS, Schuchard GH, et al. Right ventricular and right atrial collapse in patients with cardiac tamponade: a combined echocardiographic and hemodynamic study. Circulation 1984;70:966-71

12 Engel PJ, Hon H, Fowler NO, Plummer S Echocardiographic study of right ventricular wall motion in cardiac tamponade. Am 7 Cardiol 1982;50:1018-21.

13 Feinstein AR. Clinical epidimiology: the architecture of clinical research. Philadelphia: WB Saunders, 1985.

14 Armitage P, Berry G. Statistical methods in medical research. 2nd ed. Blackwell Scientific Publications, 1987.

15 Cox DR. Regression models and life tables. $\mathcal{F} R$ Stat Soc [B] 1972;34:187-220.

16 Kline IK. Cardiac lymphatic involvement by metastatic tumor. Cancer 1972;29:799-808.

17 Quraishi MA, Costanzi J, Hokanson J. The natural history of lung cancer with pericardial metastases. Cancer 1983;51:740-2.

18 Okamoto H, Shinkai T, Yamakido M, Saijo N. Cardiac tamponade caused by primary lung cancer and the management of pericardial effusion. Cancer 1993;71: 93-8.

19 Park JS, Rentschler R, Wilbur D. Surgical management of pericardial effusion in patients with malignancies. Cancer 1991;67:76-80.

20 Zipf RE, Johnston WW. The role of cytology in the evalua tion of pericardial effusions. Chest 1972;62:593-6.

21 Yazdi HM, Hajdu SI, Melamed MR. Cytopathology of pericardial effusions. Acta Cytol 1980;24:401-12.

22 Vaitkus PT, Herrmann HC, LeWinter MM. Treatment of malignant pericardial effusion. $\mathcal{f} A M A$ 1994;272: $59-64$
23 Wong B, Murphy J, Change CJ, et al. The risk of pericardiocentesis. Am $\mathcal{F}$ Cardiol 1979;44:1110-4.

24 Krikorian JG, Hancock EW. Pericardiocentesis. $\mathrm{Am} \mathcal{f} \mathrm{Med}$ 1978;65:808-14.

25 Davis S, Sharma SM, Blumberg ED, Kim CS Intrapericardial tetracycline for the management of cardiac tamponade secondary to malignant pericardial effusion. N Engl F Med 1978;299:1113-4.

26 Hawkins JW, Vacek JL. What constitutes definitive therapy of malignant pericardial effusion? "Medical" versus surgical treatment. Am Heart $₹$ 1989;118:428-32.

27 Ziskind AA, Pearce AC, Lemmon CC, et al. Percutaneous balloon pericardiotomy for the treatment of cardiac tamballoon pericardiotomy for the treatment of cardiac tamponade and large pericardial effusions: description of Cardiol 1993;21:1-5.

28 Sugimoto JT, Little AG, Ferguson MK. Pericardial window: mechanism of efficacy. Ann Thorac Surg 1990; dow: mech

29 Shepherd FA, Morgan C, Evans WK, et al. Medical management of malignant pericardial effusion by tetracycline sclerosis. Am F Cardiol 1987;60:1 161-6.

30 Palacios IF, Tuzcu EM, Ziskind AA, et al. Percutaneous balloon pericardial window for patients with malignant pericardial effusion and tamponade. Cathet Cardiovas Diagn 1991;22:244-9.

31 Chow WH, Chow TC, Cheung KL. Nonsurgical creation of a pericardial window using the Inoue balloon catheter. Am Heart f 1992;124:1100-2.

32 Jackson G, Keane D, Mishra B. Percutaneous balloon pericardiotomy in the management of recurrent malignan pericardial effusions. Br Heart $\mathcal{f}$ 1992;68:613-5.

\section{VIEWS FROM THE PAST}

\section{Cardiac museum specimens in London}

This work was funded by a modest grant from the British Heart

Foundation. The catalogue (Cardiac Museum Specimens in London) can be obtained either in printed format or as a $3 \frac{1}{2}$ inch floppy disc (IBM Compatible WordPerfect $5 \cdot 1$ ) at a cost of $£ 10$ from: Professor RH Anderson, Department

of Paediatrics, National Heart \& Lung Institute, Dovehouse Street, London SW3 6LY.
Mounted museum specimens have been used in medical teaching for over 200 years. Despite modern advances in technology and the development of more convenient and more sophisticated audiovisual teaching facilities, there is still an important role for this form of presentation-especially in cardiology, where the interpretation of modern diagnostic imaging procedures requires a high degree of familiarity with the anatomy of the heart and of the structural changes found in congenital malformations and acquired pathological conditions.

The ideal museum would contain examples of all known abnormalities and would be situated in the immediate vicinity of the place of work. This ideal does not exist. A workable alternative would be to combine, in a single catalogue, the material contained in all the museums within a finite area, so that students, teachers, research workers, and authors can know what specimens are available for study and where they can be found.

It was with this end in view that I set out on my voyages of discovery around London. My equipment consisted of a pen, a pad of paper, and my senior citizen's travel pass. There were no committee meetings, conferences, administrative procedures, or ethical debates, and the project was accomplished without the aid of computers or statistical analysis. There was, however, a lot of goodwill and unstinted cooperation from the various curators, professors, and museum technicians in all of the 17 institutions that I visited.

I had a wonderful time. I went to parts of London I had never been to before, and I viewed over 2000 pots containing examples of every conceivable form of heart disease. Rarities became commonplace after seeing seven cases of paradoxical embolus, 10 myocardial gummas, 13 "carcinoid hearts", 21 aneurysms of the sinuses of Valsalva, 40 atrial myxomas, and numerous other oddities. I saw historical treasures dating back to Napoleonic times and also many conditions that are rarely, if ever, seen in the United Kingdom. Some of the museums were themselves fascinating museum pieces especially those at $\mathrm{St}$ Bartholomew's Hospital, the Royal London Hospital, Guy's Hospital, and the Royal College of Surgeons. Sadly, some of the other collections were rather neglected.

The outcome of these visits was a listing (entitled Cardiac Museum Specimens in London) of 2188 specimens, arranged by institution and also by a comprehensive index embracing both the anatomical structures and the various types of disease processes. There is also a list of who to get in touch with at the various institutions, together with addresses, telephone numbers and the nearest Underground station.

I hope someone out there will find it useful. LEON M GERLIS Honorary Senior Lecturer, National Heart \& Lung Institute, Honorary Consultant Cardiac Pathologist, Royal Brompton Hospital 